\title{
Genetic variation among pelt sheep population using microsatellite markers
}

\author{
Shahram Nanekarani ${ }^{1 \star}$, Cyrus Amirinia ${ }^{2}$, Nour Amirmozafari ${ }^{3}$, Rasoul Vaez Torshizi ${ }^{4}$ and Ali \\ Akbar Gharahdaghi ${ }^{5}$ \\ ${ }^{1}$ Department of Animal Science, Faculty of Agriculture, Science and Research Branch, Islamic Azad University, Tehran, \\ Iran. \\ ${ }^{2}$ Department of Animal Biotechnology, Animal Science Research Institute of Iran, Karaj, Iran. \\ ${ }^{3}$ Department of Molecular Biology, Iran University of Medical Sciences, Tehran, Iran. \\ ${ }^{4}$ Animal Science Department, Faculty of Agriculture, Tarbiat Modares University, Tehran, Iran. \\ ${ }^{5}$ Department of Animal breeding, Animal Science Research Institute of Iran, Karaj, Iran.
}

Accepted 21 September, 2010

\begin{abstract}
Genetic variation in three Iranian pelt sheep breeds namely: Gray Shiraz, Zandi and Karakul were investigated using fifteen microsatellite loci. Genomic DNA was extracted from 360 blood samples by extraction kits and salting-out procedure with some modifications. The total number of alleles ranged from 6 to12 in loci. The fifteen tested loci were all polymorphic in the three breeds. The average direct count of heterozygosity overall loci in each tested breed was more than the expected heterozygosity. Tests of genotype frequencies for deviation from the Hardy-Weinberg equilibrium (HWE) were performed at each locus of overall breeds and revealed significant departure from HWE $(P<0.001)$ due to heterozygote excess. Polymorphism information content value in Gray Shiraz, Zandi and Karakul were $0.815,0.808$ and 0.808 , respectively. Rate of inbreeding within the three breeds was not noticeable (global $F_{\text {is }}=-0.19$ ). Low genetic differentiation was detected by estimation of $F_{s t}$ index between all pairs of breeds. Results showed that high level of genetic diversity was observed in pelt sheep. The phylogenetic tree based on Nei distances were drown using the neighbor-joining (NJ) and unweighted pair-group method using an arithmetic average (UPGMA). With both methods, Gray Shiraz and Karakul sheep populations were located together at one cluster and Zandi sheep population at another. The results can be useful in the development of breeding strategy for genetic improvement of pelt sheep in Iran.
\end{abstract}

Key words: Microsatellites, sheep, genetic, diversity.

\section{INTRODUCTION}

Awareness of the value of animal genetic resource is currently prompting deliberate efforts at optimum utilization and conservation of the species. The maintenance of genetic diversity is a key to the long-term survival of most species (Hall and Bardley, 1995). The genetic polymor-

*Corresponding author. E-mail: shahramnanekarani@yahoo.com. Tel: +98-261-2728772. Fax: +98-261-4413258.

Abbreviations: PIC, Polymorphism information content; PCR, polymerase chain reaction; dNTPs, deoxynucleoside triphosphates; HWE, Hardy-Weinberg equilibrium. phism and diversity found in the domestic breeds allows farmers to develop new characteristics in response to changes in environment or market conditions. So, the importance of increasing, maintaining and conserving the genetic diversity in these animals has been recognized for the future (Zhang et al., 2009). Studies of genetic diversity in domestic animals are based on an evaluation of the genetic variation within breeds and genetic relationship among them.

The total sheep population in Iran is 54 million heads. At present, a total of 1.6 million people are directly working in sheep and goat sector (ASRI, 2004). Most of sheep breeds are multipurpose producing lamb, wool and milk. Gray Shiraz, Zandi and Karakul are three wellknown breeds of sheep for pelt production in Iran with a 7438 Afr. J. Biotechnol. 
population of $500,000,500,000$ and 300,000 , respectively (ASRI, 2004). In the past, the Gray Shiraz, Karakul and Zandi were selected for production of good quality pelts mainly for exports. Now, due to the increased demand of the meat in the country, these breeds are mainly oriented towards the meat and lamb production (Moradpour, 1993). The Gray Shiraz and Karakul sheep breeds are from the same origin. Gray Shiraz has a slightly smaller size than Karakul (Tavakolian, 1999). Gray Shiraz is found in South of Iran (Fars province). Karakul is very resistant to harsh condition and is raised mostly under the semi nomadic system of management. Karakul breed is mainly found in the plain of Sarakhs in the province of Khorasan, neighboring Turkmenistan. Zandi breed is similar to Gray Shiraz and Karakul (Moradpour, 1993). It has a slightly smaller size than both two mentioned breeds. This breed is mainly found in parts of Tehran, Semnan, Ghom provinces and some parts of the Central province. These breeds are medium size, meat and pelt type, with coarse wool breed (Tavakolian, 1999). Information about population genetics is one of the most important factors in animal breeding. In order to estimate genetic diversity in the populations, molecular markers like microsatellites are useful tools (Esmaeilkhanian and Banabazi, 2006). Microsatellites are stable, polymorphic and easy to analyze; this occurs regularly throughout an animal genome as well.

Microsatellites are co-dominant markers, so that all alleles can be scored. Several studies had investigated the genetic diversity in sheep using microsatellites (EI Nahas, 2008; Dalvit et al., 2008; Mahmoudi and Babayev, 2009; Sharifi sidani et al., 2009; Kusza et al., 2010). The aim of this study is to use the molecular data to evaluate genetic variability, gene flow and inbreeding in sheep flocks, and also for designing a breeding strategy aimed at incrementing genetic diversity within and between pelt sheep breeds.

\section{MATERIALS AND METHODS}

\section{Blood sampling and DNA extraction}

Blood samples of 360 sheep of both sexes, were randomly collected from three pelt breeds: Gray Shiraz, Zandi and Karakul from distant located experimental stations belonging to the Animal Science research Institute. Genomic DNA was extracted from fresh or frozen blood using modified salting-out (Miller et al., 1988) and DNA Extraction kit (Diatom DNA Prep 100). DNA concentration was determined using a UV spectrophotometer.

\section{Microsatellite polymorphism detection}

Fifteen microsatellite markers were selected in respect of polymorphisms, a non-linkage criterion for syntenic loci and criterion of location on different chromosomes (Table 1). Microsatellites were amplified with polymerase chain reaction (PCR) using genomic DNA extracted from individual animals. The PCR was performed for each locus in $15 \mu \mathrm{l}$ reaction mixture consisting: $1 \mathrm{X}$ buffer, $200 \mu \mathrm{M}$ dNTPs, $1.5-4.5 \mathrm{mM} \mathrm{MgCl}_{2}, 0.25 \mu \mathrm{M}$ of each Primers , 1 units Taq polymerase and 100 - 200 ng DNA. The reactions were done with Gradient Master Cycler Ependorf. The cycling protocol was as follows: $5 \mathrm{~min}$ denaturing at $95^{\circ} \mathrm{C}$ followed by 35 cycles of denaturation at $95^{\circ} \mathrm{C}$ for $30 \mathrm{~s}$, annealing at $52-63^{\circ} \mathrm{C}$ (depending on primers) for $30 \mathrm{~s}$, extension at $72^{\circ} \mathrm{C}$ for $45 \mathrm{~s}$ and the final extension at $72^{\circ} \mathrm{C}$ for $5 \mathrm{~min}$. Amplification products were electrophoresed on $8 \%$ denaturing polyacrylamide gels and stained according to rapid silver staining procedure. The gel was photographed using Gel-Doc XR (BioRad). Patterns of the different genotypes for each microsatellite locus were analyzed using Gel-Pro analyzer, version 3.1 for windowsTM, which determines the allele's sizes in each animal.

\section{Statistical analysis}

Allele frequencies at each locus for each breed were calculated using a computer program GenAIEx 6 (Peakall and Smouse, 2006). GenAIEx 6 software program was also used for calculating the observed and expected heterozygosity overall loci in each of the three breeds (Levene, 1949; Nei, 1978). Test of departure from Hardy-Weinberg equilibrium at each locus overall breeds were performed using Chi-square and Likelihood ratio or G-square test (Hedrick, 2000) using GenAIEx 6 program and POPGENE software version1.31 (Yeh et al., 1999), respectively. GenAlEx 6 program was also used to calculate F-statistics that include three indices: Fit, Fst (genetic differentiation) and Fis (within breed heterozygosity deficit). Polymorphism information content (PIC) was calculated using HET software version 1.8 (Ott, 2001). The genetic distance (DS) Nei (1972) was calculated using the GenAIEx 6 program. Nei genetic distances was used to construct phylogenetic tree using the neighbor-joining method $(\mathrm{NJ})$ and the unweighted pair group method with arithmetic mean (UPGMA) using MEGA software version 3 (Kumar, 2004).

\section{RESULTS}

The number of alleles for each of the fifteen microsatellite loci in each of the three breeds is presented in Table 2. The total number of detected alleles varied from 6 (BM1815, BM1815, OARCP26, OARFCB20 and MAF64) to 12 (MCMA2 and BMS678). The mean numbers of alleles per locus are 8.1, 8 and 8.1 in Gray, Zandi and Karakul, respectively. The mean number of alleles shared between Gray and Zandi, Gray and Karakul and also Zandi and Karakul are 7.7, 7.9 and 7.8, respectively, whereas the mean number of the alleles shared by the three breeds is 7.6. It is worth mentioning that all alleles representing ten loci are present in the three breeds and the only difference found is at BMS460, OARCP26, OARAE129, MAF64 and BM6444 loci where some alleles at loci are not present in Gray, Zandi or Karakul.

Table 3 presents the alleles frequency distribution at the analyzed loci in the three breeds. At loci MCMA26, the same alleles are at the highest frequency in Gray, Zandi and Karakul breeds. Gray and Karakul breeds have similar alleles at the highest allele frequency at six loci. Whereas Gray and Zandi; Zandi and Karakul breeds show similar alleles at the highest allele frequency at three loci. Gray and Zandi breeds have the highest allele frequency at OARCP26 locus.

The average direct count of heterozygosity (observed 
Table 1. Characteristics of the microsatellites under investigation.

\begin{tabular}{|c|c|c|c|c|c|c|}
\hline Name & Primer sequence & $\begin{array}{l}\text { Chr. } \\
\text { no. }\end{array}$ & $\begin{array}{l}\text { Accession } \\
\text { no. }\end{array}$ & $\begin{array}{l}\text { Allelic } \\
\text { range }\end{array}$ & $\begin{array}{c}\text { Annealing } \\
\text { Temperature }\left({ }^{\circ} \mathrm{C}\right) \\
\end{array}$ & References \\
\hline MCMA2 & $\begin{array}{l}\text { TCACCCAACAATCATGAAAC / } \\
\text { TTAAATCGAGTGTGAATGGG }\end{array}$ & 13 & AF098773 & $157-201$ & 52 & Maddox et al., 2000. \\
\hline MCM63 & $\begin{array}{l}\text { CCCAATTTGGCAACAGCTACG / } \\
\text { ATTGGCCTCTCTCTGATGCAC }\end{array}$ & 9 & L37889 & $120-168$ & 55 & Smith et al., 1995. \\
\hline BMS460 & $\begin{array}{l}\text { TGCCCCATAGTGTAGTGCTC / } \\
\text { GCCAGCAGAGAATTGTAGCA }\end{array}$ & 3 & G18836 & $120-148$ & 58 & Maddox et al., 2000. \\
\hline BM1815 & $\begin{array}{l}\text { AGAGGATGATGGCCTCCTG / } \\
\text { CAAGGAGACAAGTCAAGTTCCC }\end{array}$ & 20 & G18389 & $\frac{\text { Not }}{\text { available }}$ & 55 & Bishop et al., 1994. \\
\hline OARCP26 & $\begin{array}{l}\text { GGCCTAACAGAATTCAGATGATGTTGC / } \\
\text { GTCACCATACTGACGGCTGGTTCC }\end{array}$ & 4 & U15698 & $120-170$ & 55 & Ede et al., 1995. \\
\hline OARFCB20 & $\begin{array}{l}\text { AAATGTGTTTAAGATTCCATACAGTG / } \\
\text { GGAAAACCCCCATATATACCTATAC }\end{array}$ & 2 & L20004 & $83-123$ & 55 & $\begin{array}{l}\text { Buchanan et al., } \\
1993 .\end{array}$ \\
\hline OARAE129 & $\begin{array}{l}\text { AATCCAGTGTGTGAAAGACTAATCCAG / } \\
\text { GTAGATCAAGATATAGAATATTTTTCAACACC }\end{array}$ & 5 & L11051 & $133-159$ & 52 & Penty et al., 1993. \\
\hline MAF64 & $\begin{array}{l}\text { AATAGACCATTCAGAGAAACGTTGAC / } \\
\text { CTCATGGAATCAGACAAAAGGTAGG }\end{array}$ & 1 & M62993 & $109-141$ & 63 & $\begin{array}{l}\text { Swarbrick et al., } \\
1991 .\end{array}$ \\
\hline BMS332 & $\begin{array}{l}\text { GACAAAACCCTTTTAGCACAGG / } \\
\text { AATTGCATGGAAAGTTCTCAGC }\end{array}$ & 22 & G18841 & $127-157$ & 57 & Maddox et al., 2000. \\
\hline LSCV38 & $\begin{array}{l}\text { GTTGCAAAGAGCTGGACGTG / } \\
\text { CTGGATGGCAAAGTGATTCAG }\end{array}$ & 12 & G40990 & $102-122$ & 54 & Maddox et al., 2000. \\
\hline BM6444 & $\begin{array}{l}\text { CTCTGGGTACAACACTGAGTCC / } \\
\text { TAGAGAGTTTCCCTGTCCATCC }\end{array}$ & 2 & G18444 & $128-165$ & 55 & Bishop et al., 1994. \\
\hline BMS995 & $\begin{array}{l}\text { AATTCTTCCAACCTCCAGTGC / } \\
\text { ACTTTTCAAGCAGGGCTCAC }\end{array}$ & 13 & G18766 & $121-147$ & 58 & Maddox et al., 2000. \\
\hline MCMA26 & $\begin{array}{l}\text { TCTCTGCTTTCCAGCCTTATTC / } \\
\text { AGAGCTTTTAGGACAGCCACC }\end{array}$ & 18 & AF098961 & $188-212$ & 52 & Maddox et al., 2000. \\
\hline BMS678 & $\begin{array}{l}\text { ACCATCTACTGTGCTATGGCTT / } \\
\text { GCAGAAACACAATACTCAGTGC }\end{array}$ & 2 & G18734 & $100-130$ & 54 & Gortari et al., 1997. \\
\hline OARCP49 & $\begin{array}{l}\text { CAGACACGGCTTAGCAACTAAACGC / } \\
\text { GTGGGGATGAATATTCCTTCATAAGG }\end{array}$ & 17 & U15702 & 85-107 & 63 & Ede et al., 1995. \\
\hline
\end{tabular}

Chr. = Chromosome; no. $=$ number. 
Table 2. Number of alleles at each microsatellite locus in the three breeds and the number of alleles shared between breeds.

\begin{tabular}{|l|c|c|c|c|c|c|c|c|}
\hline \multirow{2}{*}{ Locus } & \multicolumn{10}{|c|}{ Number of alleles } \\
\cline { 2 - 8 } & Total & Gray & Zandi & Karakul & $\begin{array}{c}\text { Shared by } \\
\text { Gray-Zandi }\end{array}$ & $\begin{array}{c}\text { Shared by } \\
\text { Gray-Karakul }\end{array}$ & $\begin{array}{c}\text { Shared by } \\
\text { Zandi-Karakul }\end{array}$ & $\begin{array}{c}\text { Shared } \\
\text { by all }\end{array}$ \\
\hline MCMA2 & 12 & 12 & 12 & 12 & 12 & 12 & 12 & 12 \\
MCM63 & 8 & 8 & 8 & 7 & 8 & 7 & 7 & 7 \\
BMS460 & 7 & 6 & 7 & 6 & 5 & 5 & 6 & 5 \\
BM1815 & 6 & 6 & 6 & 6 & 6 & 6 & 6 & 6 \\
OARCP26 & 6 & 6 & 4 & 6 & 4 & 6 & 4 & 4 \\
OARFCB20 & 6 & 6 & 6 & 6 & 6 & 6 & 6 & 6 \\
OARAE129 & 7 & 7 & 6 & 7 & 6 & 7 & 6 & 6 \\
MAF64 & 6 & 6 & 5 & 4 & 5 & 4 & 4 & 4 \\
BMS332 & 7 & 7 & 7 & 7 & 7 & 7 & 7 & 7 \\
LSCV38 & 8 & 6 & 8 & 8 & 6 & 6 & 8 & 6 \\
BM6444 & 9 & 9 & 8 & 9 & 8 & 9 & 8 & 8 \\
BMS995 & 11 & 11 & 11 & 11 & 11 & 11 & 11 & 11 \\
MCMA26 & 11 & 11 & 11 & 11 & 11 & 11 & 12 & 11 \\
BMS678 & 12 & 12 & 12 & 12 & 12 & 12 & 9 & 9 \\
OARCP49 & 9 & 9 & 9 & 9 & 9 & 9 & 7.8 & 7.6 \\
Mean & 8.3 & 8.1 & 8.0 & 8.1 & 7.7 & 7 & & \\
\hline
\end{tabular}

Table 3. Allele frequencies at each microsatellite locus in the three sheep breeds.

\begin{tabular}{|c|c|c|c|c|c|c|c|}
\hline \multirow{2}{*}{$\begin{array}{c}\text { Allele } \\
\text { no. }\end{array}$} & \multicolumn{3}{|c|}{ MCMA2 } & \multirow{2}{*}{$\begin{array}{c}\text { Allele } \\
\text { no. }\end{array}$} & \multicolumn{3}{|c|}{ BMS678 } \\
\hline & Gray & Zandi & Karakul & & Gray & Zandi & Karakul \\
\hline 1 & 0.114 & 0.062 & 0.059 & 1 & 0.063 & 0.090 & 0.174 \\
\hline 2 & 0.082 & 0.075 & 0.090 & 2 & 0.059 & 0.085 & 0.136 \\
\hline 3 & 0.091 & 0.071 & 0.069 & 3 & 0.072 & 0.056 & 0.065 \\
\hline 4 & 0.055 & 0.049 & 0.053 & 4 & 0.095 & 0.098 & 0.114 \\
\hline 5 & 0.082 & 0.058 & 0.048 & 5 & 0.050 & 0.090 & 0.054 \\
\hline 6 & 0.077 & 0.058 & 0.085 & 6 & 0.050 & 0.060 & 0.060 \\
\hline 7 & 0.068 & 0.049 & 0.074 & 7 & 0.126 & 0.124 & 0.054 \\
\hline 8 & 0.082 & 0.124 & 0.085 & 8 & 0.081 & 0.051 & 0.049 \\
\hline 9 & 0.141 & 0.080 & 0.122 & 9 & 0.108 & 0.115 & 0.054 \\
\hline 10 & 0.059 & 0.137 & 0.090 & 10 & 0.113 & 0.081 & 0.065 \\
\hline 11 & 0.077 & 0.142 & 0.112 & 11 & 0.108 & 0.060 & 0.076 \\
\hline 12 & 0.073 & 0.097 & 0.112 & 12 & 0.077 & 0.090 & 0.098 \\
\hline \multirow{2}{*}{$\begin{array}{c}\text { Allele } \\
\text { no. }\end{array}$} & \multicolumn{3}{|c|}{ MCM63 } & \multirow{2}{*}{$\begin{array}{c}\text { Allele } \\
\text { no. }\end{array}$} & \multicolumn{3}{|c|}{ LSCV38 } \\
\hline & Gray & Zandi & Karakul & & Gray & Zandi & Karakul \\
\hline 1 & 0.045 & 0.081 & 0.128 & 1 & 0.000 & 0.144 & 0.128 \\
\hline 2 & 0.067 & 0.242 & 0.267 & 2 & 0.000 & 0.051 & 0.059 \\
\hline 3 & 0.067 & 0.119 & 0.133 & 3 & 0.117 & 0.051 & 0.064 \\
\hline 4 & 0.147 & 0.085 & 0.067 & 4 & 0.230 & 0.292 & 0.239 \\
\hline 5 & 0.179 & 0.199 & 0.211 & 5 & 0.157 & 0.157 & 0.096 \\
\hline 6 & 0.183 & 0.161 & 0.133 & 6 & 0.052 & 0.051 & 0.053 \\
\hline 7 & 0.121 & 0.064 & 0.000 & 7 & 0.261 & 0.203 & 0.250 \\
\hline 8 & 0.192 & 0.051 & 0.061 & 8 & 0.183 & 0.051 & 0.112 \\
\hline \multirow{2}{*}{$\begin{array}{c}\text { Allele } \\
\text { no. }\end{array}$} & \multicolumn{3}{|c|}{ OARAE129 } & Allele & \multicolumn{3}{|c|}{ BMS332 } \\
\hline & Gray & Zandi & Karakul & no. & Gray & Zandi & Karakul \\
\hline 1 & 0.050 & 0.284 & 0.066 & 1 & 0.118 & 0.173 & 0.121 \\
\hline 2 & 0.073 & 0.208 & 0.055 & 2 & 0.236 & 0.261 & 0.253 \\
\hline
\end{tabular}


Table 3. Contd.

\begin{tabular}{|c|c|c|c|c|c|c|c|}
\hline 3 & 0.055 & 0.127 & 0.093 & 3 & 0.264 & 0.235 & 0.275 \\
\hline 4 & 0.161 & 0.161 & 0.181 & 4 & 0.086 & 0.111 & 0.082 \\
\hline 5 & 0.275 & 0.169 & 0.181 & 5 & 0.145 & 0.084 & 0.104 \\
\hline 6 & 0.206 & 0.051 & 0.159 & 6 & 0.082 & 0.084 & 0.115 \\
\hline 7 & 0.179 & 0.000 & 0.264 & 7 & 0.068 & 0.053 & 0.049 \\
\hline \multirow{2}{*}{$\begin{array}{c}\text { Allele } \\
\text { no. }\end{array}$} & \multicolumn{3}{|c|}{ BM1815 } & \multirow{2}{*}{$\begin{array}{c}\text { Allele } \\
\text { no. }\end{array}$} & \multicolumn{3}{|c|}{ OARFCB20 } \\
\hline & Gray & Zandi & Karakul & & Gray & Zandi & Karakul \\
\hline 1 & 0.197 & 0.118 & 0.304 & 1 & 0.140 & 0.291 & 0.134 \\
\hline 2 & 0.272 & 0.193 & 0.380 & 2 & 0.331 & 0.261 & 0.274 \\
\hline 3 & 0.145 & 0.218 & 0.065 & 3 & 0.182 & 0.145 & 0.226 \\
\hline 4 & 0.154 & 0.160 & 0.065 & 4 & 0.144 & 0.107 & 0.075 \\
\hline 5 & 0.127 & 0.261 & 0.082 & 5 & 0.093 & 0.090 & 0.118 \\
\hline 6 & 0.105 & 0.050 & 0.103 & 6 & 0.110 & 0.107 & 0.172 \\
\hline \multirow{2}{*}{$\begin{array}{c}\text { Allele } \\
\text { no. }\end{array}$} & \multicolumn{3}{|c|}{ OARCP26 } & \multirow{2}{*}{$\begin{array}{c}\text { Allele } \\
\text { no. }\end{array}$} & \multicolumn{3}{|c|}{ MAF64 } \\
\hline & Gray & Zandi & Karakul & & Gray & Zandi & Karakul \\
\hline 1 & 0.049 & 0.114 & 0.137 & 1 & 0.134 & 0.325 & 0.397 \\
\hline 2 & 0.433 & 0.452 & 0.170 & 2 & 0.196 & 0.213 & 0.196 \\
\hline 3 & 0.357 & 0.333 & 0.335 & 3 & 0.165 & 0.254 & 0.326 \\
\hline 4 & 0.063 & 0.101 & 0.181 & 4 & 0.335 & 0.163 & 0.082 \\
\hline 5 & 0.049 & 0.000 & 0.099 & 5 & 0.085 & 0.046 & 0.000 \\
\hline 6 & 0.049 & 0.000 & 0.077 & 6 & 0.085 & 0.000 & 0.000 \\
\hline \multirow{2}{*}{$\begin{array}{c}\text { Allele } \\
\text { no. }\end{array}$} & \multicolumn{3}{|c|}{ BMS995 } & \multirow{2}{*}{$\begin{array}{c}\text { Allele } \\
\text { no. }\end{array}$} & \multicolumn{3}{|c|}{ MCMA26 } \\
\hline & Gray & Zandi & Karakul & & Gray & Zandi & Karakul \\
\hline 1 & 0.077 & 0.141 & 0.130 & 1 & 0.047 & 0.057 & 0.104 \\
\hline 2 & 0.128 & 0.098 & 0.089 & 2 & 0.060 & 0.070 & 0.066 \\
\hline 3 & 0.068 & 0.103 & 0.120 & 3 & 0.085 & 0.110 & 0.115 \\
\hline 4 & 0.098 & 0.051 & 0.052 & 4 & 0.175 & 0.162 & 0.159 \\
\hline 5 & 0.081 & 0.090 & 0.073 & 5 & 0.145 & 0.149 & 0.066 \\
\hline 6 & 0.120 & 0.077 & 0.130 & 6 & 0.098 & 0.079 & 0.088 \\
\hline 7 & 0.077 & 0.073 & 0.052 & 7 & 0.051 & 0.070 & 0.099 \\
\hline 8 & 0.068 & 0.090 & 0.068 & 8 & 0.073 & 0.057 & 0.066 \\
\hline 9 & 0.068 & 0.098 & 0.125 & 9 & 0.094 & 0.061 & 0.055 \\
\hline 10 & 0.077 & 0.051 & 0.052 & 10 & 0.120 & 0.101 & 0.066 \\
\hline 11 & 0.137 & 0.128 & 0.109 & 11 & 0.051 & 0.083 & 0.115 \\
\hline \multirow{2}{*}{$\begin{array}{c}\text { Allele } \\
\text { no. }\end{array}$} & \multicolumn{3}{|c|}{ BM6444 } & \multirow{2}{*}{$\begin{array}{c}\text { Allele } \\
\text { no. }\end{array}$} & \multicolumn{3}{|c|}{ OARCP49 } \\
\hline & Gray & Zandi & Karakul & & Gray & Zandi & Karakul \\
\hline 1 & 0.174 & 0.058 & 0.050 & 1 & 0.133 & 0.098 & 0.059 \\
\hline 2 & 0.071 & 0.096 & 0.050 & 2 & 0.150 & 0.107 & 0.128 \\
\hline 3 & 0.192 & 0.233 & 0.111 & 3 & 0.168 & 0.171 & 0.117 \\
\hline 4 & 0.089 & 0.175 & 0.150 & 4 & 0.164 & 0.197 & 0.122 \\
\hline 5 & 0.121 & 0.254 & 0.150 & 5 & 0.053 & 0.051 & 0.170 \\
\hline 6 & 0.089 & 0.067 & 0.083 & 6 & 0.093 & 0.051 & 0.096 \\
\hline 7 & 0.063 & 0.075 & 0.122 & 7 & 0.093 & 0.150 & 0.053 \\
\hline 8 & 0.116 & 0.042 & 0.161 & 8 & 0.053 & 0.090 & 0.112 \\
\hline \multirow[t]{5}{*}{9} & 0.085 & 0.000 & 0.122 & 9 & 0.093 & 0.085 & 0.144 \\
\hline & & Allele no. & \multicolumn{3}{|c|}{ BMS460 } & & \\
\hline & & & Gray & Zandi & Karakul & & \\
\hline & & 1 & 0.000 & 0.143 & 0.000 & & \\
\hline & & 2 & 0.000 & 0.237 & 0.043 & & \\
\hline
\end{tabular}


Table 3. Contd.

\begin{tabular}{|l|l|l|l|l|l|l|l|}
\hline & 3 & 0.127 & 0.147 & 0.085 & & \\
& & 4 & 0.145 & 0.116 & 0.197 & & \\
& & 0.219 & 0.112 & 0.234 & & \\
& & 0.219 & 0.174 & 0.319 & & \\
& & 0.149 & 0.071 & 0.122 & & \\
& & 0.140 & 0.000 & 0.000 & & \\
\hline
\end{tabular}

The highest allele frequency/breed is in bold typeface. no. = Number.

Table 4. Mean heterozygosity and polymorphism information content (PIC) in the three sheep breeds.

\begin{tabular}{|l|c|c|c|}
\hline \multicolumn{1}{|c|}{ Heterozygosity and PIC } & Gray & Zandi & Karakul \\
\hline Mean observed heterozygosity \pm SD & $0.9841 \pm 0.0297$ & $0.9857 \pm 0.0152$ & $0.9885 \pm 0.0117$ \\
Mean expected heterozygosity \pm SD & $0.8366 \pm 0.0619$ & $0.8297 \pm 0.0654$ & $0.8306 \pm 0.0643$ \\
Mean PIC \pm SD & $0.8155 \pm 0.0743$ & $0.8077 \pm 0.0803$ & $0.8081 \pm 0.0780$ \\
\hline
\end{tabular}

SD, Standard deviation.

Table 5. PIC, $F_{\mathrm{IT}}, \mathrm{F}_{\mathrm{ST}}, \mathrm{G}_{\mathrm{ST}}$ and $\mathrm{F}_{\mathrm{IS}}$ values, and chi-square and $\mathrm{G}$-square test for HWE for each locus over all breeds.

\begin{tabular}{|c|c|c|c|c|c|c|c|c|}
\hline LOCUS & PIC & $F_{I T}$ & $F_{\text {ST }}$ & $F_{\text {IS }}$ & $\mathrm{G}_{\mathrm{ST}}$ & Chi-square & G-square & Df \\
\hline MCMA2 & 0.906 & -0.075 & 0.005 & -0.080 & 0.005 & $653.2^{\star * *}$ & $618.3^{\star * \star}$ & 66 \\
\hline MCM63 & 0.842 & -0.158 & 0.021 & -0.183 & 0.021 & $402.6^{\star \star *}$ & $394.2^{\star \star *}$ & 28 \\
\hline BMS460 & 0.828 & -0.172 & 0.034 & -0.213 & 0.034 & $558.6^{\star \star \star}$ & $467.6^{\star \star *}$ & 28 \\
\hline BM1815 & 0.786 & -0.216 & 0.030 & -0.253 & 0.030 & $302.9^{\star \star \star}$ & $300.3^{* \star *}$ & 15 \\
\hline OARCP26 & 0.680 & -0.361 & 0.032 & -0.406 & 0.032 & $726.2^{\star \star \star}$ & $444.7^{\star * *}$ & 15 \\
\hline OARFCB20 & 0.781 & -0.219 & 0.011 & -0.233 & 0.011 & $222.2^{\star \star \star}$ & $266.5^{\star \star \star}$ & 15 \\
\hline OARAE129 & 0.830 & -0.167 & 0.042 & -0.218 & 0.042 & $483.2^{\star \star \star}$ & $477.3^{\star \star \star}$ & 21 \\
\hline MAF64 & 0.743 & -0.224 & 0.039 & -0.274 & 0.039 & $359.9^{\star \star \star}$ & $333.4^{* * *}$ & 15 \\
\hline BMS332 & 0.796 & -0.190 & 0.003 & -0.193 & 0.003 & $247.5^{\star \star *}$ & $278.3^{\star * *}$ & 21 \\
\hline LSCV38 & 0.807 & -0.196 & 0.013 & -0.212 & 0.013 & $600.6^{\star * *}$ & $513.5^{\star \star \star}$ & 15 \\
\hline BM6444 & 0.859 & -0.137 & 0.019 & -0.159 & 0.019 & $424.7^{\star \star \star}$ & $407.1^{\star \star \star}$ & 36 \\
\hline BMS995 & 0.896 & -0.082 & 0.004 & -0.086 & 0.004 & $275.1^{\star * *}$ & $268.5^{\star * *}$ & 55 \\
\hline MCMA26 & 0.891 & -0.111 & 0.005 & -0.117 & 0.005 & $676^{* \star *}$ & $619^{* * *}$ & 55 \\
\hline BMS678 & 0.906 & -0.082 & 0.007 & -0.090 & 0.007 & $634.9^{* * *}$ & $588.4^{* * *}$ & 78 \\
\hline OARCP49 & 0.869 & -0.127 & 0.010 & -0.139 & 0.010 & $617.7^{\star \star \star}$ & $585.7^{\star \star *}$ & 36 \\
\hline Mean & 0.828 & -0.168 & 0.018 & -0.19 & 0.018 & & & \\
\hline
\end{tabular}

*** $p<0.001$.

heterozygosity) overall loci in Gray, Zandi and Karakul breeds are $0.984,0.986$ and 0.988 , respectively. Whereas the average expected heterozygosity overall loci in the three breeds are $0.837,0.830$ and 0.831 , respectively (Table 4). These results show more heterozygosity than expected in each breed. Mean PIC overall loci in Gray, Zandi and Karakul breeds are 0.8155, 0.8077 and 0.8081 , respectively (Table 4 ). As expected, fairly high level of genetic heterogeneity was further reflected within three breeds by a mean PIC value. These high estimates of PIC substantiated the suitability of used set of markers to applications such as parentage control, linkagemapping programs in addition to genetic polymorphism studies in Iranian sheep too.

The PIC values for all loci ranged from 0.680 (OARCP26) to 0.906 (MCMA2). The PIC values are very high, indicating that these loci are highly informative and suitable for genetic studies of sheep breeds (Table 5).

Tests of genotype frequencies for deviation from Hardy-Weinberg equilibrium (HWE), at each locus overall breeds, reveal significant departure from HWE $(P>$ 0.001) (Table 5). $\mathrm{G}_{\mathrm{ST}}$ and F-statistics for each locus overall 
Table 6. Per pair Fst values between all pairs of the tested breeds.

\begin{tabular}{|l|c|c|}
\hline Breed & Gray & Zandi \\
\hline Gray & & \\
\hline Zandi & 0.013 & \\
Karakul & 0.013 & 0.015 \\
\hline
\end{tabular}

Table 7. Nei's genetic distances between the studied breeds.

\begin{tabular}{|l|c|c|}
\hline Breed & Gray & Zandi \\
\hline Gray & & \\
Zandi & 0.141 & \\
Karakul & 0.139 & 0.155 \\
\hline
\end{tabular}

breeds are given in Table 5. The global $F_{I S}, F_{S T}$ and $F_{I T}$ are $-0.19,0.018$ and -0.168 , respectively. All markers had negative values of $F_{I S}$, showing an excess of heterozygotes. $F_{S T}$ values of genetic differentiation and GST values of breed differentiation were similar and ranged from 0.003 (BMS332) to 0.042 (OARAE129). Per pair estimator of FST, which is the measure of differentiation among population, is 0.013 between Gray and Zandi, 0.013 between Gray and Karakul and 0.015 between Zandi and Karakul (Table 6). The calculated genetic distance matrix is shown in Table 7. The distance between Gray and Zandi, and Gray and Karakul was smaller than the distance between Zandi and Karakul. Nei' (1978) genetic distances among breeds are presented in Table 7, with the corresponding UPGMA dendrogram and neighbour-joining tree in Figures 1 and 2, respectively. The smallest genetic distance $(0.139)$ was estimated between Gray and Karakul. There is little differentiation between the pelt sheep breeds with Nei distances ranging from 0.139 to 0.155 . The cluster analysis shows that Gray and Karakul breeds cluster independently from Zandi breed.

\section{DISCUSSION}

Recently, the preservation of unique, genetically distinct breeds of domesticated animals, especially indigenous, has received much attention. Knowledge and information on genetic diversity, population structure and genetic relationships between populations are absolute prerequisites for defining and accomplishing effective preservation strategies (Kusza et al., 2010). This study aimed to characterize the genetic diversity and structure of pelt sheep populations by using fifteen microsatellites. Over the past decade, numerous studies on genetic diversity in domestic livestock (mainly in small ruminants), based on the analysis of microsatellite loci, have been carried out worldwide. Investigation of genetic variation in
Taleshi goat using microsatellite loci indicated substantial genetic variation based on their gene diversity and average number of alleles per locus (Mahmoudi and Babayev, 2009). Genetic characterization of Alpine sheep breeds was established on the basis of individual genotypes at microsatellite loci (Dalvit et al., 2008). Study of genetic relationships among bulgarian sheep breeds using microsatellite loci indicated a high level of variation in the tested breeds (Kusza et al., 2010). Arora et al. (2008) examines the genetic variability in Jalauni, an important sheep of northwestern arid and semi arid region of India, at 25 microsatellite loci. Also, genetic diversity and relationships within and among Magra, Marwari and Sonadi sheep breeds of India, were distinguished based on microsatellite markers (Arora et al., 2008).

In Iran, many studies were performed to evaluate the genetic diversity of Iranian sheep breeds. Esmail et al. (2007) used nineteen microsatellites to evaluate genetic variation within Baluchi sheep breed. Banabazi et al. (2007) studied the genetic variation within and between five Iranian sheep populations including Sanjabi, Kordi Kordistan, Kordi Khorasan, Mehraban and Moghani using six microsatellite Markers. Also, genetic variation among different ecotypes of the Iranian sanjabi sheep was investigated based on the analysis of microsatellite loci (Sharifi et al., 2009). In the present study, fifteen microsatellite loci were used to evaluate the genetic diversity within and between pelt sheep breeds reared in Iran. The fifteen microsatellites are all polymorphic in the three breeds. The total number of alleles was 122 at the 15 studied loci. Major differences between the three breeds were not observed. The average expected heterozygosity overall loci in Gray, Zandi and Karakul are 0.837, 0.830 and 0.831 , respectively. High value of average expected heterozygosity within the breed could be attributed to the large allele numbers detected in the tested loci (Kalinwski, 2002). The average direct count of heterozygosity overall loci in each of the three sheep breeds is more than the expected heterozygosity.

All loci were derived from Hardy-Weinberg equilibrium $(p<0.001)$ due to excess of heterozygote individuals than homozygote individuals, migration, high mutation rate in microsatellite and artificial selection in all breeds (Aminafshar et al., 2008). Deviation from HWE at microsatellites loci have, also been reported in various studies (Barker et al., 2001; Laval et al., 2000; El Nahas, 2008; Aminafshar et al., 2008; Sharifi sidani et al., 2009). It is known that a population is considered to be within HWE only when it is able to maintain its relative allele frequencies.

The global inbreeding coefficients $F_{I S}(-0.19)$ and $F_{I T}$ (0.168 ) observed in the present study indicate an excess of heterozygotes and so it does not probably encounter problems that results from inbreeding depression. This result may explain the observed high value of direct count of heterozygosity in each breed and the deviation from HWE which were detected in all loci overall breeds. 


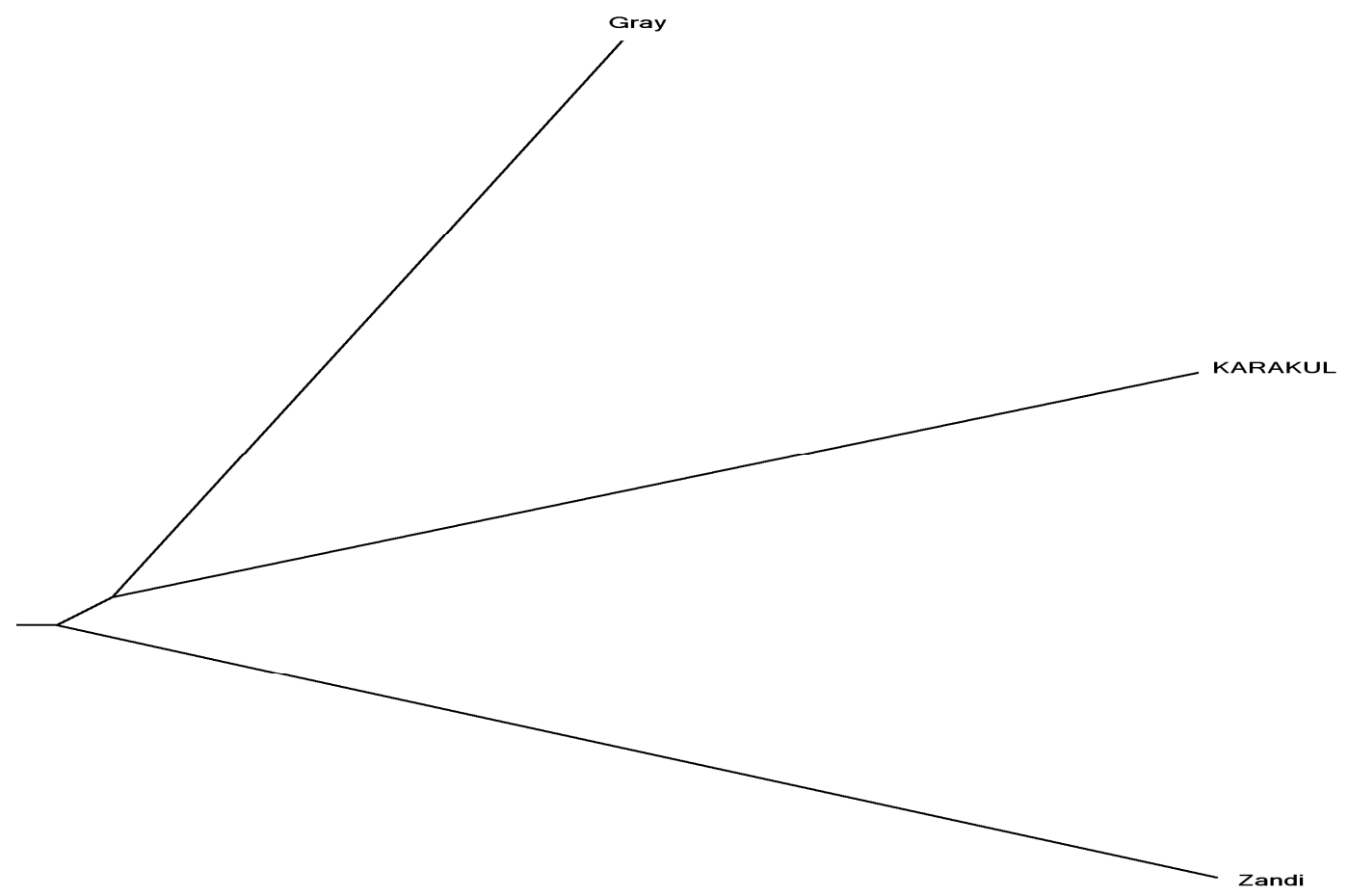

Figure 1. UPGMA phylogenetic radial tree based on Nei's standard genetic distances of the three sheep breeds.

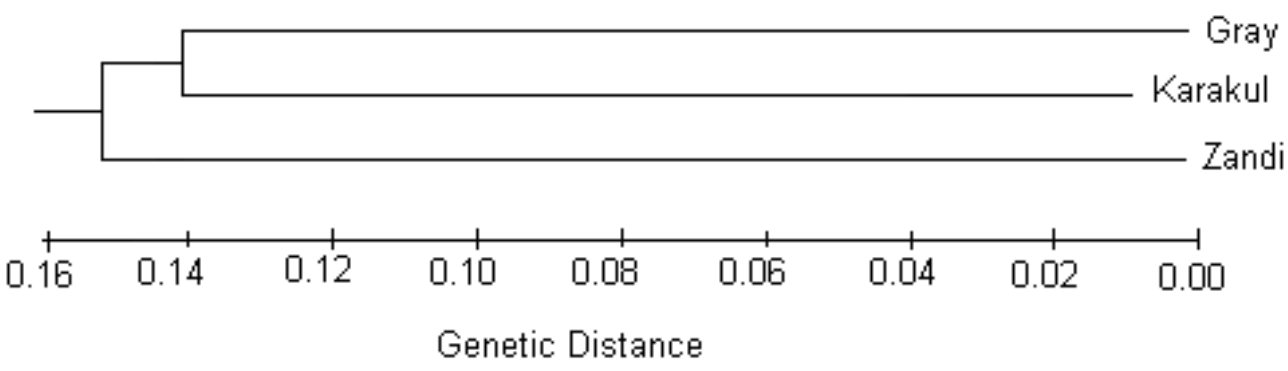

Figure 2. Neighbour-joining phylogenetic tree based on Nei's standard genetic distances.

According to Hartl (1980), per pair $\mathrm{F}_{\mathrm{ST}}$ value equals 0.05 is indicative for moderate differentiation between populations. The per pair FST values reported in the present investigation between all pairs of the tested breeds are less than 0.05 which may indicate a low differentiation between populations under investigation. The estimated $F_{S T}$, which corresponds to the proportion of genetic variability accounted for by differences among breeds, was 0.018 . These results indicate that genetic diversity quantified by microsatellite markers shows very little differentiation among pelt sheep breeds. Our results are similar to those reported for other sheep breeds, where $F_{S T}$ estimates range between 0.03 and 0.08 (Arranz et al., 1998; Alvarez et al., 2004; Rendo et al., 2004; Sodhi et al., 2006; Peter et al., 2007; El Nahas et al., 2008). The genetic similarity observed among three breeds is probably a result of migration among populations that may have a common origin and which have been selected mostly for morphological traits associated with the breed standard. Migration has a great effect on the reduction of genetic differentiation between popula-tions (Laval et al., 2000). Additionally, low genetic distance values $(\mathrm{Ds}=0.139-0.155)$ supported high genetic similarity between these three breeds and were in similar range with those cited by Arranz et al. (1998) and Sodhi et al. (2006) for closely related Spanish sheep breeds (Ds $=0.21-0.36$ ) and Nali and Chokla sheep (Ds $=0.229$ ), respectively. In summary, the three breeds were found to be genetically and closely related to each other, although there was a significant geographical distance between the three populations.

In conclusion, the tested microsatellites, being all polymorphic in the three breeds, could be fruitfully used for the differentiation between breeds. The evaluation of 
genetic variations within and between pelt sheep breeds may be used as basis for the development of common breeding strategy for genetic improvement of these breeds.

\section{REFERENCES}

Alvarez I, Royo LJ, Fernandez I, Gutierrez JP, Gomez E, Goyache F (2004). Genetic relationships and admixture among sheep breeds from Northern Spain assessed using microsatellites. J. Anim. Sci. 82: 2246-2252.

Aminafshar M, Amirinia C, Torshizi RV (2008). Genetic diversity in buffalo population of guilan using microsatellite marker. J. Anim. Vet. Adv. 7(11): 1499-1502.

Animal Science Research Institute of IRAN Tehran (2004). Draft Iran's Country Report On Farm Animal Genetic Resources.Ministry of Jihad-e-Agriculture.

Arora R, Bhatia S, Sehrawat A, Maity SB, Kundu SS (2008). Genetic variability in Jalauni sheep of India inferred from microsatellite data. National Bureau of Animal Genetic Resources, P.O. Box 129, Karnal132001, Haryana, India.

Arranz JJ, Bayón Y, San Primitivo F (1998). Genetic relationships among Spanish sheep using microsatellites, Anim. Genet. 29: 435440.

Banabazi MH, Esmaeilkhanian S, Miraei Ashtiani SR, Moradi Shahrbabak M (2007). Gentic variation within and between five iranian sheep populations using microsatellite markers. (B), Isf. Univ. Technol., Isf., Iran. J. Sci. Technol. Agric. Nat. Res. 10: p. 4.

Barker JSF, Tan SG, Moore SS, Mukherjee Tk, Matheson JL, Selvaraj OS (2001). Genetic variation within and relationship among populations of Asian goats (Capra hircus). J. Anim. Breed. Genet. 118: 213-233.

Dalvit C, Sacca E, Cassandro M, Gervaso M, Pastore E, Piasentier E (2008). Genetic diversity and variability in Alpine sheep breeds. Small Rum. Res. 80: 45-51.

El Nahas SM, Hassan AA, Abou Mossallam AA, Mahfouz ER, Bibars MA, Oraby HAS, de Hondt HA (2008). Analysis of genetic variation in different sheep breeds using microsatellites. Afr. J. Biotechnol. 7(8): 1060-1068.

Esmail KS, Nejati JA, Afraz F, Daneshyar P, Ghanbari S (2007). Genetic variation among baluchi sheep population using microsatellite markers. J. (B), Isf. Univ. Technol., Isf., Iran. Sci. Tech. Agric. Nat. Res. 11: p. 41.

Hall SJG, Bradley DG (1995). Conserving livestock breed biodiversity. Trends Ecol. Evol. 10: 267-270.

Hartl D (1980). Principles of population genetics. Sinauer Associates Inc, Sunderland Massachussets, USA.

Hedrick PW (2000). Genetics of Populations 2nd Ed, Boston: Jones and Bartlett.

Kalinwski ST (2002). How many alleles per locus should be used to estimate genetic distances. Heredity, 88: 62-65.

Kumar S, Tamura K, Nei M (2004). MEGA3: Integrated software for Molecular Evolutionary Genetics Analysis and sequence alignment. Briefings in Bioinform, 5: 150-163.
Kusza SD, Dimov I, Nagy Z, Bõsze A, Jávor S, Kukovics (2010). Microsatellite analysis to estimate genetic relationships among five bulgarian sheep breeds. Genet. Mol. Biol. 33: p. 1.

Laval G, Iannuccelli N, Legault C, Milan D, Groenen MAM, Giuffra E, Andersson L, Nissen PH, Jorgensen CB, Beeckmann P, Geldermann H, Foulley JL, Chevalet C, Ollivier L (2000). Genetic diversity of eleven European pig breeds. Genet. Select. Evol. 32: 187-203.

Levene $H$ (1949). On a matching problem in genetics. Ann. Math. Statist. 20: p. 91

Mahmoudi B, Babayev MS (2009). The investigation of genetic variation in Taleshi goat using microsatellite marker. Res. J. Biol. Sci. 4(6): 644-646.

Miller SA, Dykes DD, Polesky HF (1988). A simple salting out procedure for extracting DNA from human nucleated cells. Nucleic Acids Res. 16: p. 1215.

Moradpour R (1993). Introduction to Karakul Shiraz, Organization of Jahad-e-Sazandegi of Fars province. (In Persian).

Nei M (1972). Genetic distance between populations. Am. Nat. 106: 283-292.

Nei M (1978). Estimation of average heterozygosity and genetic distance from a small number of individuals. Genetics, 89: 583-590.

Ott J (2001). Program HET Version 1.8. Utility programs for analysis of genetic linkage. Rockefeller University.New York, NY, USA.

Peakall R, Smouse PE (2006). GENALEX 6 : genetic analysis in Excel. Population genetic software for teaching and research. Mol. Ecol. Note, 6: 288-295.

Peter C, Bruford M, Perez T, Dalamitra S, Hewitt G, Erhardt, G, The Econogene Consortium (2007). Genetic diversity and subdivision of 57 European and Middle Eastern sheep breeds. Anim. Genet. 38: 3744.

Rendo F, Iriondo M, Jugo BM, Mazón LI, Aguirre A, Vicario A, Estonba A (2004). Tracking diversity and differentiation in six sheep breeds from the North Iberian Peninsula through DNA variation. Small Rum. Res. 52: 196-202.

Sharifi sidani E, Amirinia C, Lavaf A, Farasati C, Aminafshar M (2009). Genetic variation among different ecotypes of the iranian sanjabi sheep. J. Anim. Vet. Adv. 8(6): 1173-1176.

Sodhi M, Mukesh M, Bhatia S (2006). Characterizing Nali and Chokla sheep differentiation with microsatellite markers. Small Rum. Res. 65: 185-192.

Tavakolian J (1999). An outlook toward the livestock and poultry genetic resources of Iran, Animal Sciences Research Institute, Karaj, Iran.

Yeh FC, Boyle T, Yang R (1999). POPGENE version 1.31. A Microsoft window based freeware for population genetic analysis. University of Alberta, Edmonton.

Zhang XY, Zhou ML, Zhang XH, Wu DJ (2009). Study on population genetic structure of Liangshan semi-wool sheep using microsatellite markers. 